\title{
Gedankenaustausch zum 90sten Geburtstag von Frau Prof. Ursula Schmidt-Tintemann: Was soll Plastische Chirurgie? Was soll sie nicht?
}

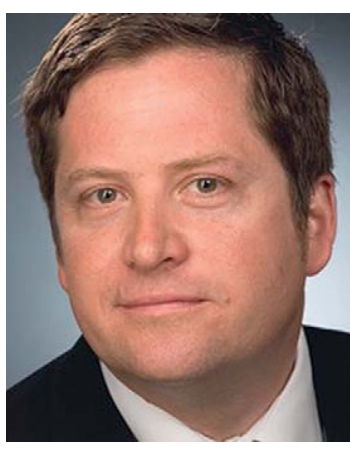

Univ.-Prof. Dr. med. Riccardo E. Giunta
Liebe Leserinnen und Leser,

im Juni diesen Jahres feierte Frau Prof. Ursula Schmidt-Tintemann ihren 90sten Geburtstag. Sie erfreut sich sehr guter Gesundheit und hat ihren Geburtstag im kleinen Kreise gefeiert. Darüber hinaus haben wir als Handchirurgie, Plastische Chirurgie, Ästhetische Chirurgie der LudwigMaximilians Universität München zu diesem schönen Anlass ein Symposium veranstaltet über welches in diesem Editorial berichtet werden soll (๑ Abb. 1).

Frau Prof. Schmidt-Tintemann hat Ende der 50er Jahre die Abteilung für Plastische Chirurgie am Klinikum rechts der Isar als eine der ersten Einheiten in Deutschland begründet. Zum 50. Jubiläum der Abteilung in 2008 hatten wir ihre Pionierleistungen in dieser Zeitschrift zusammengefasst [1]. Neben der umfangreichen Aufbauarbeit im Klinikum hat sie als eine der 4 Gründungsmitglieder gemeinsam mit Prof. Rudolf Zellner, Prof. Dieter Buck-Gramcko und Prof. Fritz Müller die Vereinigung der Deutschen Plastischen Chirurgen (VDPC), der jetzigen Deutschen Gesellschaft der Plastischen, Rekonstruktiven und Ästhetischen Chirurgen (DGPRÄC) begründet.

\section{Ernst von Siemens Auditorium der Pinakothek der Moderne \\ $\nabla$}

Mit dem Ernst von Siemens Auditorium der Pinakothek der Moderne München fanden wir rasch einen sehr schönen Veranstaltungsort, der dem Ereignis in vielerlei Hinsicht gerecht wurde. $\mathrm{Zu}$ aller erst greift der künstlerische Aspekt in der modernen Kunst sehr schön die hohen Ansprüche der Plastischen Chirurgie im Hinblick auf Ästhetik des Geformten auf. Das Privileg des Plastischen Chirurgen ist es, in gewissen Grenzen auch kreativ zu sein. Mit einem modernen Auditorium fanden wir auch alle notwendigen technischen Voraussetzungen. Die Veranstaltung hat erfreulicherweise enormen Zuspruch gefunden, sodass letztlich mehr als 250 Personen gezählt wurden ( $\bullet$ Abb. 2). Darunter sehr viele Professoren, auch aus unterschiedlichen Fachbereichen und Regionen Deutschlands.

\section{„Was soll Plastische Chirurgie? Was soll sie nicht?" $\nabla$}

Als Thema der Veranstaltung haben wir die von Frau Prof. Schmidt-Tintemann und dem Autor dieses Editorials vor nahezu einem Jahr aufgeworfenen Fragen „Was soll Plastische Chirurgie? Was soll sie nicht?“ [2] gewählt. Die auf den ersten Blick sehr einfach formulierte Frage hat bei genauerer Betrachtung eine unerwartete Tiefe und Bedeutung für das Fachgebiet.

Das Konzept des Symposiums bestand in einem Gedankenaustausch zu diesem Thema aus verschiedenen Sichtweisen. Herausragende Experten beleuchteten sowohl die wichtigsten historischen und inhaltlichen Aspekte in der Entwicklung des Fachgebiets Plastische Chirurgie als auch die aktuelle Situation und haben Ausblicke und Perspektiven des Fachgebiets für die Zukunft entwickelt.

Unter den Referenten waren der Präsident der Deutschen Gesellschaft für Chirurgie und ehemaliger Präsident der DGPRÄC Peter Vogt, der die Bedeutung der Plastischen Chirurgie als vollwertige, eigenständige „Säule“ innerhalb der chirurgischen Fachgebiete dargestellt hat. Die Sichtweise auf die strukturelle Weiterentwicklung der Chirurgie hat der ehemalige Generalsekretär der Deutschen Gesellschaft für Chirurgie und Viszeralchirurg, Hartwig Bauer, exzellent zusammengefasst. Einen sehr schönen historischen Blick aus Sicht eines Plastischen Chirurgen der Gründerzeit hat Hanno Millesi aus Wien beigetragen. Wir möchten gerade ihm besonders danken, da er keine Mühen gescheut und die Reise nach München auf sich genommen hat. Jutta Liebau hat als Präsidentin der DGPRÄC nicht nur die Fachgesellschaft vertreten, sondern auch das Engagement von Frau Prof. Schmidt-Tintemann für die Rolle der Frau in der Chirurgie gewürdigt.

\section{Heinz Maier Leibniz-Medaille $\nabla$}

Die Festrede wurde erfreulicherweise vom Präsidenten der Technischen Universität München, Prof. Dr. Dr. h.c. mult. Wolfgang A. Herrmann übernommen, der durch den Ärztlichen Direktor des Klinikums rechts der Isar, Herrn Prof. Reiner Gradinger, vertreten war. Neben der Leistung für die Abteilung Plastische Chirurgie am Klinikum rechts der Isar und für das Fachgebiet wurde besonders der Beitrag von Frau Prof. Schmidt-Tintemann in der intellektuellen Werteentwicklung und Konzipierung des Fachgebiets und ihre persönliche Bedeutung als ethisch-moralische Instanz in der Plastischen Chirurgie gewürdigt. Gleichzeitig wurde auch das Engagement für die sich wandelnde Rolle der Frau in der klassischen Männerdomäne Chirurgie gelobt. Als Frau Prof. 


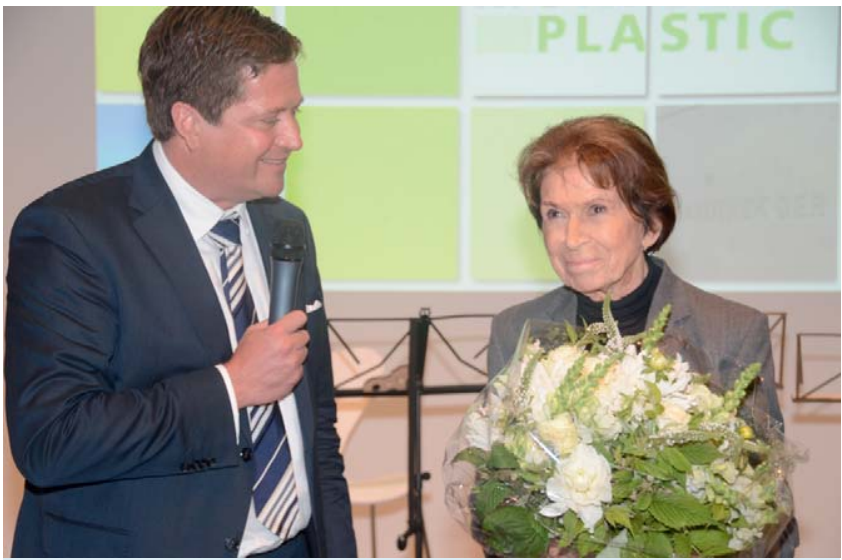

Abb. 1 Frau Prof. Ursula Schmidt-Tintemann mit dem Autor beim Symposium zum 90sten Geburtstag in der Pinakothek der Moderne mit dem Veranstalter. (Quelle: R. E. Giunta, Ludwig-Maximilians Universität München)

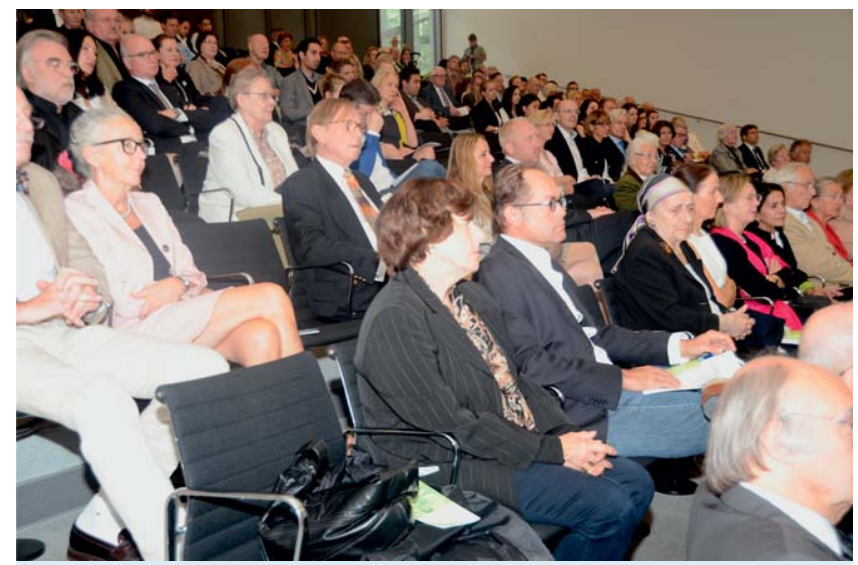

Abb. 2 Auditorium der Pinakothek der Moderne. (Quelle: R. E. Giunta, Ludwig-Maximilians Universität München)
Schmidt-Tintemann 1969 die Venia legendi erhielt gab es an der Technischen Hochschule München eine einzige berufene Professorin. Mittlerweile lehren mehr als 80 Professorinnen an der TU München. Herr Gradinger hat als Repräsentant der TU München Frau Prof. Schmidt-Tintemann in Würdigung Ihrer Leistungen die Heinz Maier Leibniz-Medaille verliehen.

Im Anschluss wurden die aufgeworfenen Fragen von den Schülern von Frau Prof. Schmidt-Tintemann, die wichtige Leitungsfunktionen in den unterschiedlichsten Regionen Deutschlands übernommen haben, für die einzelnen inhaltlichen Aspekte dargestellt und beantwortet. Allesamt haben die Ideen der Plastischen Chirurgie weitergetragen und selbst weiterentwickelt. Sie haben in Leitungsfunktionen ihre Gedanken bereits an die übernächste Generation weitergegeben und damit selbst Entscheidendes für das Fachgebiet beigetragen.

In diesem Teil traten die Referenten in weitgehend in alphabetischer Reihenfolge auf. Edgar Biemer machte als direkter Nachfolger am Klinikum rechts der Isar den Anfang und berichtete über die Mikrochirurgie und ihre große Bedeutung in der Rekonstruktion nach Tumor bzw. Trauma. Im direkten Anschluss berichte Axel Feller, der selbst über Jahre eine große Abteilung für Plastische Chirurgie in Vogatreuth geleitete hatte, über die Entwicklung Brustrekonstruktion. Beide waren Lehrer des Autors dieses Editorials, sodass ich dankbar die Ideen aufgenommen habe und in meiner derzeitigen Leitungsfunktion wiederum an die nachfolgenden Generationen weiter geben möchte.

Daran anschließend berichtete Rüdiger Olbrisch über Plastische Brustchirurgie, die er lange Jahre als Chefarzt der Plastischen Chirurgie in Düsseldorf vertreten hat. Wolfgang Mühlbauer hätte über viele Themen umfassend sprechen können, hat aber das Thema Kraniofaziale Chirurgie gewählt und seine beeindruckenden Langzeitfälle bei angeborenen Fehlbildungen dargestellt. Der langjährige Leiter der Plastischen Chirurgie in

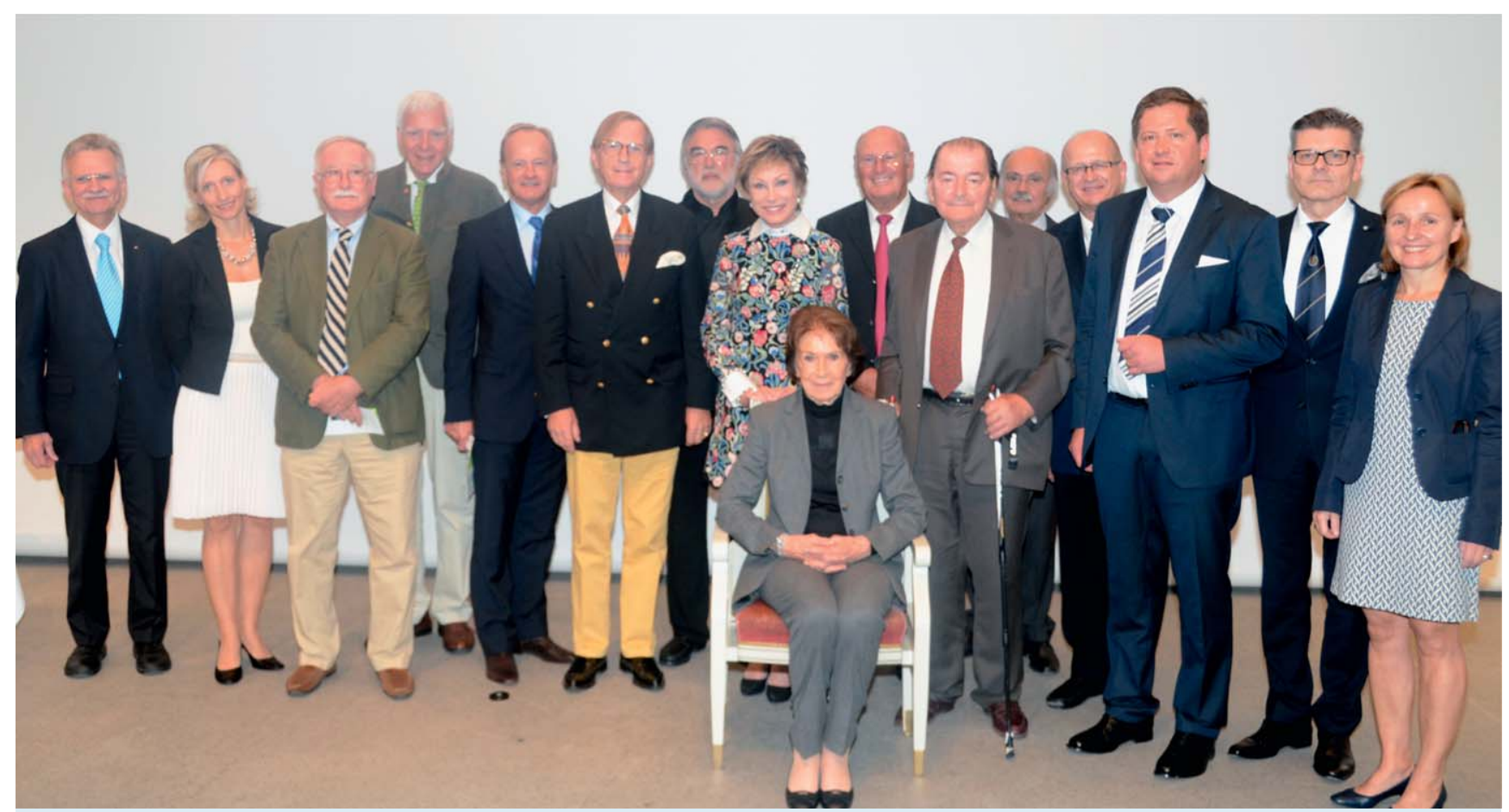

Abb. 3 Jubilarin Frau Prof. Ursula Schmidt-Tintemann (im Sessel der Bayerischen Nationaloper aus den 60er Jahren) mit den Vortragenden und Referenten von links nach rechts: Prof. Reiner Gradinger, Frau Dr. Julia Schmid, Prof. Giulio Ingianni, Prof. Rüdiger Olbrisch, Prof. Axel.Mario Feller, Prof. Edgar Biemer, Dr. Heinz Schöneich, Frau Dr. Antje-Katrin Kühnemann, Prof. Wolfgang Mühlbauer, Prof. Hanno Millesi, Prof. Hartwig Bauer, Prof. Ulrich Steinau, Prof. Riccardo Giunta, Prof. Peter Vogt, Frau Prof. Jutta Liebau. (Quelle: R. E. Giunta, Ludwig-Maximilians Universität München) 
Wuppertal und Miteinlader zur Veranstaltung, Giulio Ingianni, berichtete über seine Erfahrungen in der Plexuschirurgie. Mit einer eindrücklichen Videopräsentation fasste Heinz Schöneich die Aktivitäten von Interplast zusammen. Ulrich Steinau, selbst erster Plastischer Chirurg als Präsident der DGCH und Senator auf Lebenszeit der Gesellschaft sowie ehemaliger Präsident der DGPRÄC zeigte die Bedeutung der Plastischen Chirurgie in der Rekonstruktion nach Tumoren auf. Längst hat auch in der Tumorchirurgie die Lebensqualität für den Patienten nach Tumorresektion eine hohe Bedeutung erlangt, sodass die Plastische Chirurgie als festes Mitglied in der interdisziplinären Behandlung von Tumoren längst nicht mehr weg zu denken ist. Den Abschluss bildete Christoph Wolfensberger aus Zürich, der über die Möglichkeiten der Ästhetischen Chirurgie berichtete.

Moderiert wurde dieser Teil neben dem Veranstalter auch von Frau Antje-Katrin Kühnemann, die sich als Moderatorin der vom Bayerischen Fernsehen produzierten Sendung „Die Sprechstunde - Ratschläge für die Gesundheit“ vom Anfang der 70er Jahre bis 2007 für die Patientenaufklärung, verdient gemacht hat. Nachdem Frau Kühnemann in den 70er Jahren einige Monate unter Frau Prof. Schmidt-Tintemann als Assistenzärztin gearbeitet hatte, hatte sie einen persönlichen Bezug zur Jubilarin und zur Plastischen Chirurgie entwickelt.

Allen Referenten, Beitragenden, den Sponsoren und Spendern sowie meinem Team an der Ludwig-Maximilians Universität München möchte ich für ihre Unterstützung in der Vorbereitung der Veranstaltung danken ( $\bullet$ Abb. 3.). Besonders gedankt werden soll dem Jugend Cello "Quartetto Crescendo“, welches mit der musikalischen Eröffnung und dem Abschluss einen sehr schönen Bogen zur Jugend spannen konnte.
In der Zusammenschau der schönen Veranstaltung zu einem wichtigen Thema möchte ich resümieren, dass es wichtig ist, Ereignisse wie den 90sten Geburtstag von Frau Prof. Schmidt-Tintemann auch in dieser Form zu begehen und damit an die Ursprünge der Plastischen Chirurgie in Deutschland zu erinnern. Die zahlreichen Teilnehmer der jüngeren Generationen haben gezeigt, dass es sehr wohl auch junge engagierte Talente gibt, die künftig das Fachgebiet weiter entwickeln werden. Ein erfolgreicher Brückenschlag an die nachfolgenden Generationen ist verständlicherweise ein großes wichtiges Anliegen der älteren Generation. Es besteht durchaus Hoffnung, dass das Fachgebiet auch in Zukunft weitere Impulse erhält und sich erfolgreich weiter entwickeln wird.

Zuletzt möchte ich Frau Prof. Schmidt-Tintemann danken: sie hat der Veranstaltung nach unserer sorgfältigen Abwägung der Chancen und Risiken zugestimmt und hat sie in jeder Hinsicht unterstützt. Trotz ihres Lebensalters hat sie die Mühen und Anstrengungen nicht gescheut, eine solche, für die Plastische Chirurgie wichtige Veranstaltung zu organisieren. Ihr Engagement für Ideen und Werte wird mir immer ein großes Vorbild sein.

\section{Literatur}

1 Giunta RE. Ursula-Schmidt-Tintemann - Pionierin der Plastischen Chirurgie in Deutschland. Handchir Mikrochir Plast Chir 2008; 40: 408-410

2 Giunta RE, Schmidt-Tintemann U. Was soll Plastischer Chirurgie? Was soll sie nicht? Handchir Mikrochir Plast Chir 2013; 45: 191-192 